\title{
Editorial
}

\section{Iterative Methods and Dynamics for Nonlinear Problems}

\author{
Y. I. Kim, ${ }^{1}$ A. Cordero, ${ }^{2}$ J. R. Sharma, ${ }^{3}$ F. Soleymani, ${ }^{4}$ J. R. Torregrosa, ${ }^{2}$ and X. Wang ${ }^{5}$ \\ ${ }^{1}$ Dankook University, Cheonan, Republic of Korea \\ ${ }^{2}$ Universitat Politècnica de València, Valencia, Spain \\ ${ }^{3}$ Sant Longowal Institute of Engineering \& Technology, Punjab, India \\ ${ }^{4}$ Ferdowsi University of Mashhad, Mashhad, Iran \\ ${ }^{5}$ Bohai University, Liaoning, China \\ Correspondence should be addressed to Y. I. Kim; yikbell@yahoo.co.kr
}

Received 9 October 2017; Accepted 11 October 2017; Published 16 November 2017

Copyright (C) 2017 Y. I. Kim et al. This is an open access article distributed under the Creative Commons Attribution License, which permits unrestricted use, distribution, and reproduction in any medium, provided the original work is properly cited.

Since the advent of Kung-Traub's conjecture established in 1974 on optimal convergence of iterative memory-free methods, the development of iterative methods and their dynamics has been focused for several decades until present times to improve the order of convergence, initial condition behavior, and CPU time for effectively solving nonlinear equations encountered in many fields of sciences such as applied mathematics, computational physics, engineering mathematics, and nonlinear discrete dynamics and chaos. Real-life nonlinear problems including weather forecast, satellite-orbit tracing, image processing via discrete data, and locating objects through global positioning systems can be solved by efficient iterative methods with the aid of highprecision modern computers.

The primary aim of this special issue is for authors from diversified scientific disciplines to accomplish their high-quality research goals that seek recent developments and innovational techniques on iterative methods and their dynamics for nonlinear problems under consideration. The areas to be covered in this issue are root-finding iterative methods for nonlinear equations, nonlinear recurrence relations, fixed point theory, initial-boundary value problems, stability analysis and dynamics of iteration functions, and computational complexity of iterative techniques.

In total, 34 papers were initially submitted in this special issue, covering various aspects of iterative or dynamical approaches to resolve their governing equations of the system. After thorough inspections and microscopic strict reviews on these papers, it is our regret to be able to select only five papers for their publication in the current issue, even though many of them were of very good quality. Those selected five papers describe very important topics dealing with iterative maps from functional analysis, root-finding Padé-like approximants, recurrence relations from climate data, recursive optimization on modern traffic control systems, and dynamical analysis from partial differential equations reducing carbon emission based on strict government regulations.

\section{Acknowledgments}

Our special gratitude must be expressed to enthusiastic participants in preparation of their manuscripts as well as to contributing reviewers of these manuscripts from the international community.

Y. I. Kim

A. Cordero

J. R. Sharma

F. Soleymani

J. R. Torregrosa

$X$. Wang 


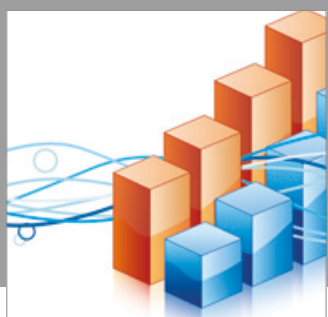

Advances in

Operations Research

vatersals

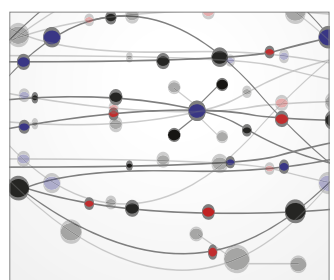

\section{The Scientific} World Journal
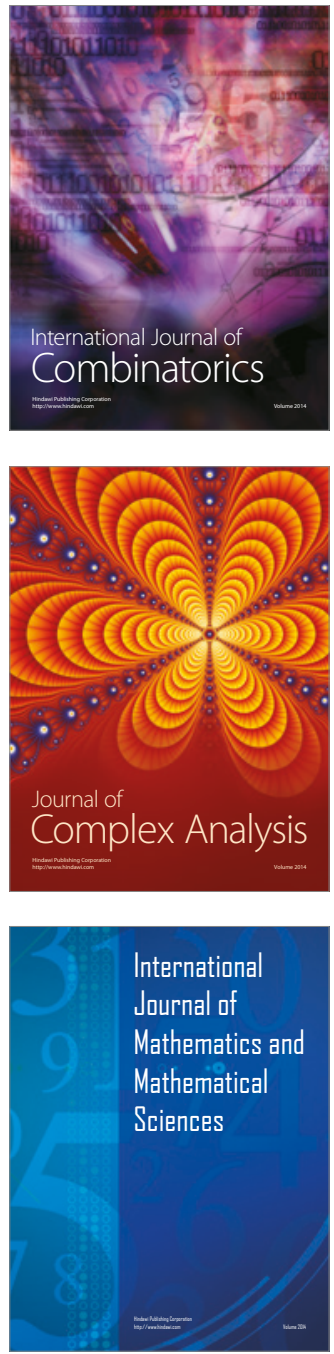
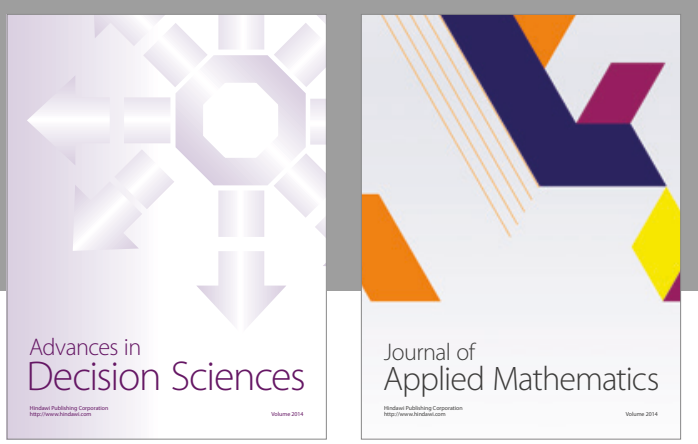

Algebra

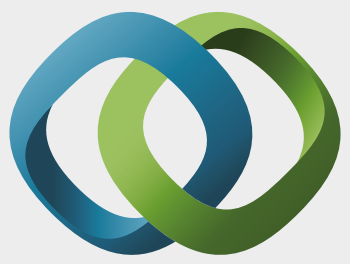

\section{Hindawi}

Submit your manuscripts at

https://www.hindawi.com
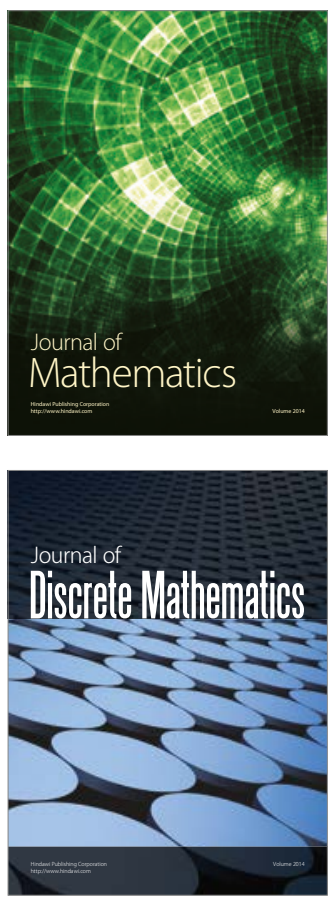

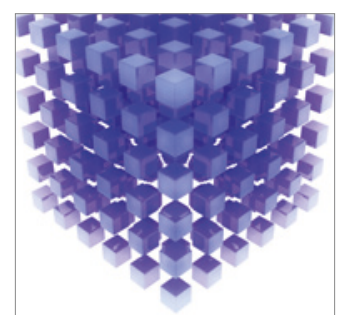

Mathematical Problems in Engineering
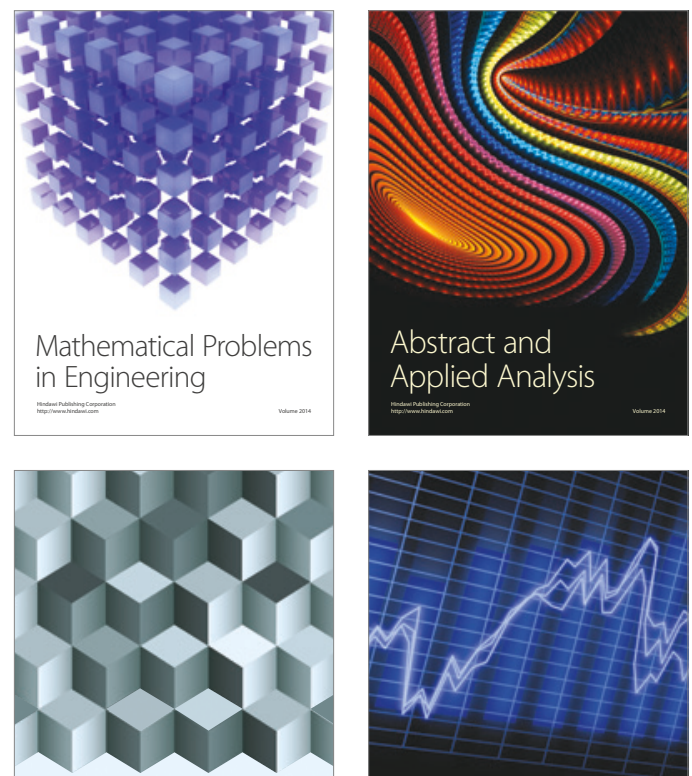

Journal of

Function Spaces

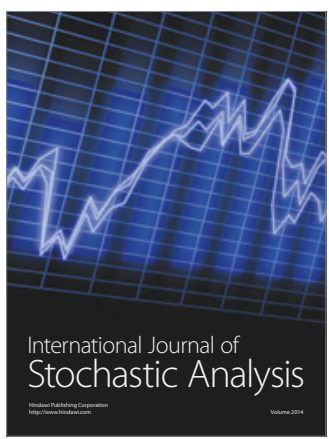

Probability and Statistics
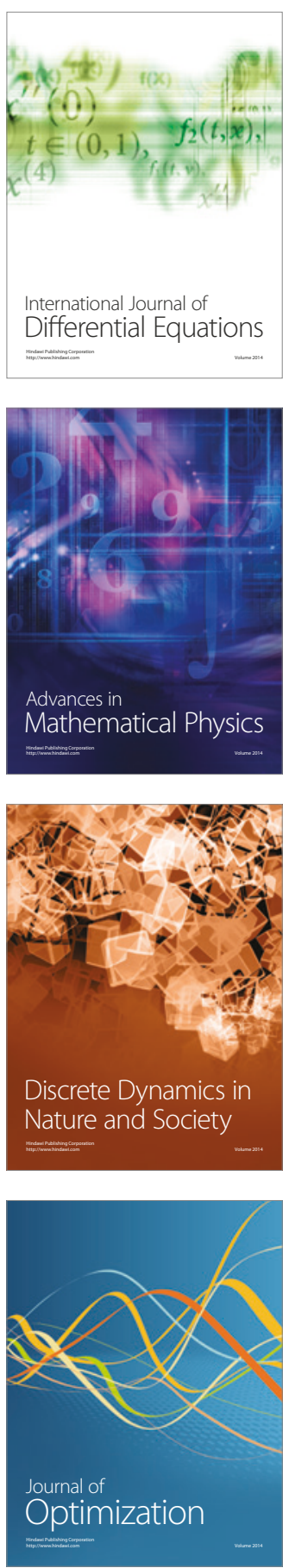